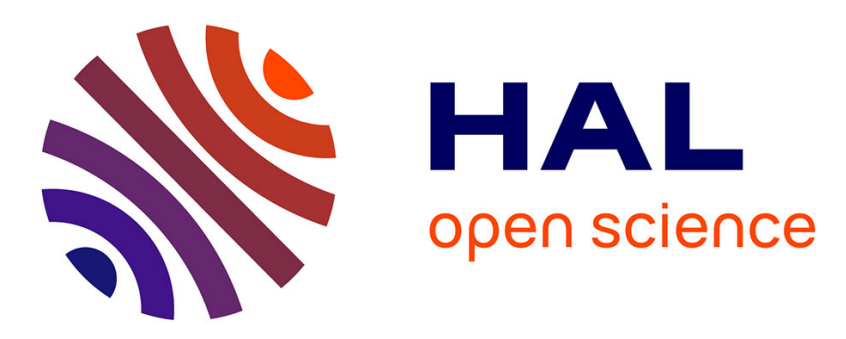

\title{
Motion Compensation In Digital Holography For Retinal Imaging
}

Julie Rivet, Guillaume Tochon, Serge Meimon, Michel Paques, Thierry

Géraud, Michaël Atlan

\section{- To cite this version:}

Julie Rivet, Guillaume Tochon, Serge Meimon, Michel Paques, Thierry Géraud, et al.. Motion Compensation In Digital Holography For Retinal Imaging. 2019 IEEE 16th International Symposium on Biomedical Imaging (ISBI), Apr 2019, Venice, Italy. pp.1428-1431, 10.1109/ISBI.2019.8759564 . hal-02183084

\section{HAL Id: hal-02183084 \\ https://hal.science/hal-02183084}

Submitted on 15 Jul 2019

HAL is a multi-disciplinary open access archive for the deposit and dissemination of scientific research documents, whether they are published or not. The documents may come from teaching and research institutions in France or abroad, or from public or private research centers.
L'archive ouverte pluridisciplinaire HAL, est destinée au dépôt et à la diffusion de documents scientifiques de niveau recherche, publiés ou non, émanant des établissements d'enseignement et de recherche français ou étrangers, des laboratoires publics ou privés. 


\title{
MOTION COMPENSATION IN DIGITAL HOLOGRAPHY FOR RETINAL IMAGING
}

\author{
Julie Rivet ${ }^{1,2}$ Guillaume Tochon ${ }^{1}$ Serge Meimon ${ }^{3}$ Michel Paques $^{4}$ Thierry Géraud $^{1}$ Michael Atlan $^{2}$ \\ ${ }^{1}$ EPITA Research and Development Laboratory (LRDE) \\ ${ }^{2}$ ESPCI Paris, PSL Research University, Sorbonne Université, CNRS, Institut Langevin \\ ${ }^{3}$ ONERA - the French Aerospace Lab \\ ${ }^{4}$ Institut de la Vision, CIC 1423, INSERM UMR-S 968, CNRS, Sorbonne Université
}

\begin{abstract}
The measurement of medical images can be hindered by blur and distortions caused by the physiological motion. Specially for retinal imaging, images are greatly affected by sharp movements of the eye. Stabilization methods have been developed and applied to state-of-the-art retinal imaging modalities; here we intend to adapt them for coherent light detection schemes. In this paper, we demonstrate experimentally crosscorrelation-based lateral and axial motion compensation in laser Doppler imaging and optical coherence tomography by digital holography. Our methods improve lateral and axial image resolution in those innovative instruments and allow a better visualization during motion.
\end{abstract}

Index Terms - Motion Compensation, Cross-correlation, Digital Holography, Optical Coherence Tomography, Laser Doppler Holography.

\section{INTRODUCTION}

Ophtalmology is an ancient science which has benefited from technological progresses in optics. The first images of eye fundus in vivo by holographic ophtalmoscopy $[1,2]$ paved the way to the development of time-averaged holography for retinal vessels imaging. This non-invasive and low-exposure optical interferometric imaging technology has made accessible the retinal imaging, leading to better diagnosis of several diseases like glaucoma, macular degeneration and diabetes. Later, retinal blood flow contrasts were also successfully rendered by narrowband [3] and wideband [4] digital holography.

Digital holography relies on the acquisition and the processing of interference images created by the superposition of two light waves from the same source. The first wave is a reference, whereas the second is back-scattered by the retina. Several facets of digital holography have been developped during the past few years to take maximum advantage of phase variations between both waves which contain numerous information about the retina. Optical Coherence Tomography (OCT) [5, 6] permits optical sectioning of retinal layers. On the other hand, laser Doppler imaging [7] focuses on the detection of local dynamics.

Digital holography faces several issues altering images quality. Eye motion during image acquisition distorts the signal. In order to stabilize images of retinal features, image registration can be used. It consists in finding the geometric transformation to align two images. Medical image registration techniques have been developped during the past years [8]. Although most of the literature in this field concerns radiological modalities, several researches have been led in retinal imaging. The most widely used technique is featurebased registration of eye fundus images $[9,10]$. However in our case, feature-based registration is not suitable because objects of interest can disappear or even be replaced by another structure if the movement of the eye is too large, which could bias the search of feature points. This article is focused on cross-correlation stabilization to compensate lateral and axial motion of the whole image structure in laser Doppler holography and swept-source holographic OCT, respectively.

\section{HOLOGRAPHIC IMAGING}

\subsection{Setup}

Optical interferograms are recorded with a laser Doppler instrument [3] in human eyes, and with a holographic OCT instrument in synthetic phantoms. For the laser Doppler setup, the source is a laser diode emitting at the wavelength $\lambda=785$ $\mathrm{nm}$. The camera records $2048 \times 2048$-pixel images corresponding to the $(x, y)$ plane, at a frame rate of $\omega_{\mathrm{S}} /(2 \pi)=80$ $\mathrm{Hz}$ with 8 bit/pixel quantization. For OCT, the beam is emitted by a tunable laser and varies from $\omega_{1}$ to $\omega_{2}$, which are linked to the wavelengths $\lambda_{1}=870 \mathrm{~nm}$ and $\lambda_{2}=820 \mathrm{~nm}$ by $\omega=2 \pi c / \lambda$, where $c$ is the speed of light, with a sweep time $T=0.5 \mathrm{~s}$. The camera records $1024 \times 1024$-pixel images at a frame rate of $\omega_{\mathrm{S}} /(2 \pi)=512 \mathrm{~Hz}$ with $16 \mathrm{bit} /$ pixel quantization. Digitized interferograms from the camera are processed in real-time with the Holovibes ${ }^{1}$ software to compute and visualize holograms, and saved for offline processing.

\subsection{Acquisition of interferograms}

A Mach-Zehnder interferometer has been used to record interferogams. It consists in making interfere two beams from the same laser source. The source is split between reference and object arms. The light wave from the object arm is backscattered once the sample is reached and intereferes in the camera

\footnotetext{
1http://holovibes.com
} 
plane with the beam in the reference arm. The interferogram $I$ acquired in the camera plane $(x, y)$ at time $t$ is expressed as:

$$
I(x, y, t)=\left|E(x, y, t)+E_{\mathrm{LO}}(x, y, t)\right|^{2},
$$

where the object field is $E$ and the reference field is $E_{\mathrm{LO}}$. The phase difference between both optical fields is noted $\phi$. The off-axis configuration of the setups allows a spatial separation between the constant term, and the cross-beating interferometric contribution, which will produce the image of interest, and its conjugate [11] (see Fig. 2a).

\subsection{Processing of optically-acquired interferograms}

Interferogram rescaling with wavelength. In order to avoid the distortion of the signal, the impact of the sweep in OCT needs to be considered to propagate the interferograms $I$ from the camera plane to the retina or image plane. In fact the size of the pixels in the image plane depends on the wavelength of the beam and of the distance between camera and image planes [12,13]: $d^{\prime}=\lambda z /(N d)$, where $d$ and $d^{\prime}$ are the lateral size of a pixel from camera plane and from image plane, respectively, $z$ is the distance between both planes and $N$ is the number of pixels in one lateral dimension. Because of the sweep of the source, the pixels of the image plane shrink with wavelength (arrow (1) of Fig. 1). To circumvent lateral field variation with wavelength, each interferogram is resampled by linear interpolation of the calculation grid with a different pitch (arrow (2) of Fig. 1). The rescaled interferogram is:

$$
I^{\prime}(x, y, t)=I\left(x \lambda / \lambda_{1}, y \lambda / \lambda_{1}, t\right),
$$

where $\lambda$ is the current wavelength. In the case of laser Doppler imaging, the optical wavelength $\lambda$ is kept constant, hence the interferogram does not need to be rescaled: $I^{\prime} \equiv I$.

Spatial demodulation by Fresnel transform. The propagation of the fields from camera to image plane is carried out by a Fresnel transform [11] (arrow (3) of Fig. 1), which gives the hologram $H(x, y, t)$ :

$$
\begin{aligned}
& H(x, y, t)=\frac{i}{\lambda z} \exp (-i k z) \iint I^{\prime}\left(x^{\prime}, y^{\prime}, t\right) \\
& \times \exp \left[\frac{-i \pi}{\lambda z}\left(\left(x-x^{\prime}\right)^{2}+\left(y-y^{\prime}\right)^{2}\right)\right] \mathrm{d} x^{\prime} \mathrm{d} y^{\prime} .
\end{aligned}
$$

The argument of the complex-valued hologram in the crosscontribution region is the phase difference $\phi$ between the optical fields $E$ and $E_{\mathrm{LO}}$ in the image plane.

Temporal demodulation by Fourier transform. In sweptsource OCT, the instantaneous beating frequency $\partial \phi / \partial t=\omega$ scales up linearly with axial depth $z$ [5], whereas in laser Doppler, it describes local velocities of the scatterers [7]. Hence for both methods, temporal signal demodulation is performed by short-time Fourier transform (STFT) (arrow (4) of Fig. 1). Time-and-space-dependent spectrograms

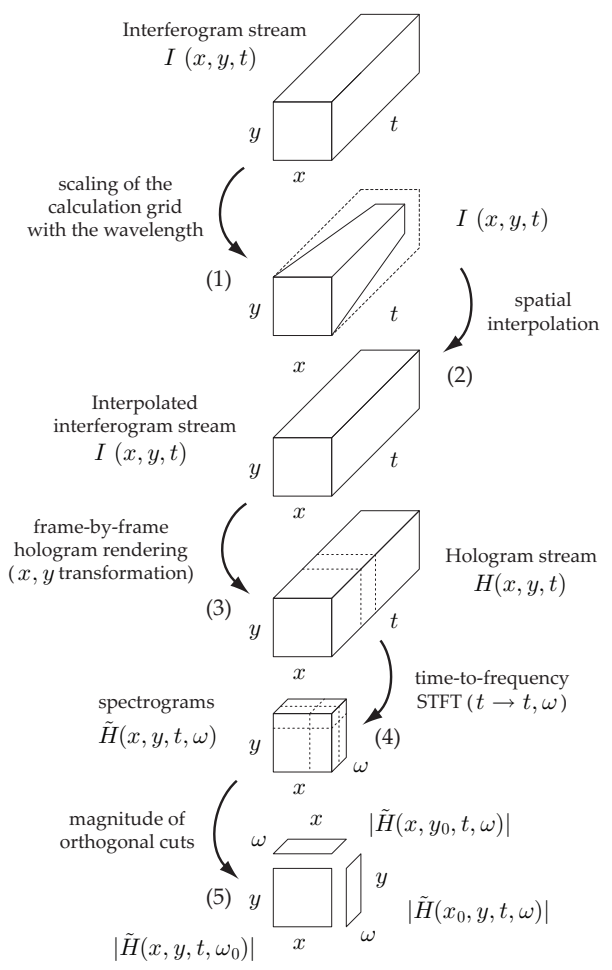

Fig. 1: Sketch of the process of image propagation. The raw data in the camera plane are transferred in the image plane and are expressed with depth.

$\tilde{H}(x, y, t, \omega)$ are calculated from the stream of intermediate holograms $H(x, y, t)$ :

$$
\tilde{H}(x, y, t, \omega)=\int H(x, y, \tau) g_{T}(t-\tau) e^{-i \omega \tau} \mathrm{d} \tau,
$$

where $g_{T}(t)$ is a time gate of width $T$ at time $t$. Then, the envelope $|\tilde{H}|$ of the STFT of $H$ is formed (arrow (5) of Fig. 1). The quantities $|\tilde{H}(x, y, t, \omega)|$ are the images in laser Doppler and OCT on which motion compensation will be performed.

\section{MOTION COMPENSATION METHODS}

\subsection{Lateral Motion Compensation}

We form Doppler-contrast images at the Nyquist frequency $\omega_{\mathrm{S}} / 2$ by calculating Eq. (4) with a time gate $g_{T}$ of two points ( $T=25 \mathrm{~ms}$ ). To compensate lateral motion (in $(x, y)$ plane), we use a cross-correlation-based stabilization algorithm. The human eye is constantly subject to deviations, saccades and tremors. Added to heartbeat and respiration motion, those movements can shift or even remove the object of interest from the image during several frames. Thus, a processing chain has been built in order to keep important structures in the center of the en-face image.

A reference image $f_{1}$ is made by averaging $n=10$ consecutive images, and compared to a moving average $f_{2}$ of 


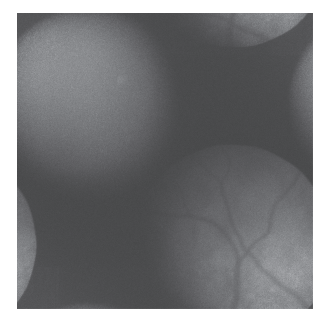

(a) Off-axis digital hologram

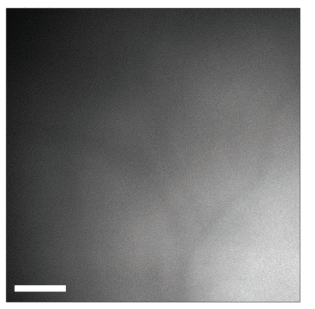

(c) Time-average of raw images

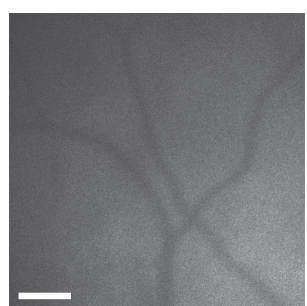

(b) Region of interest

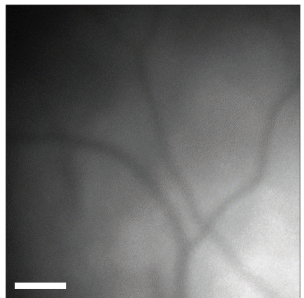

(d) Time-average of stabilized images

Fig. 2: Retinal image stabilization by lateral $(x, y)$ motion compensation of intensity holograms (Section 3.1). The white scale bars indicate $200 \mu \mathrm{m}$.

$n=10$ images in the neighborhood of the current image:

$$
\begin{aligned}
& f_{1}(x, y)=\sum_{t=1}^{n}\left|\tilde{H}\left(x, y, t, \omega_{\mathrm{S}} / 2\right)\right| / n, \\
& f_{2}(x, y)=\sum_{t=1}^{n}\left|\tilde{H}\left(x, y, t+\tau, \omega_{\mathrm{S}} / 2\right)\right| / n .
\end{aligned}
$$

The quantitative comparison is performed with a normalized and centered cross-correlation [14]. The difference between the position of the maximum value and the center of the correlation matrix $\gamma$ gives the displacement between the two images. Resulting shifts describe retina lateral motion.

\subsection{Axial Motion Compensation}

We form OCT images by calculating Eq. (4) with a time gate $g_{T}$ of 256 points $(T=0.5 \mathrm{~s})$. Axial motion during the recording process has a negative impact on the reconstruction of OCT images: when an axial drift occurs, the depth information encoded in the beating frequency of the interferogram is inaccurate, adding an offset phase to the signal and decreasing in-depth accuracy. The principle of the compensation method is to identify where the phase shift due to the axial motion occurs by using STFT [15] on 20-point windows. Indeed, a global axial displacement of the sample during acquisition can be spotted in the time Fourier domain, where it corresponds to a frequency shift.

For $y=y_{0}$, the reference image $f_{1}$ and the sliding image $f_{2}$ are defined as the modulus of the STFT of $H\left(x, y_{0}, t\right)$ whose sub-windows are fixed or moving, respectively:

$$
\begin{aligned}
& f_{1}(x, \omega)=\left|\int H\left(x, y_{0}, \tau\right) g_{T}\left(t_{0}^{\prime \prime}-\tau\right) e^{-i \omega \tau} \mathrm{d} \tau\right|, \\
& f_{2}(x, \omega)=\left|\int H\left(x, y_{0}, \tau\right) g_{T}\left(t_{0}^{\prime}-\tau\right) e^{-i \omega \tau} \mathrm{d} \tau\right|,
\end{aligned}
$$

where $g_{T}$ is a 20-point Hanning window, and $t_{0}^{\prime}$ and $t_{0}^{\prime \prime}$ are the centers of the moving and the reference windows, respec-

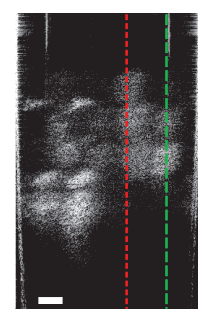

(a)

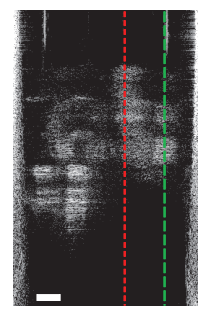

(b)

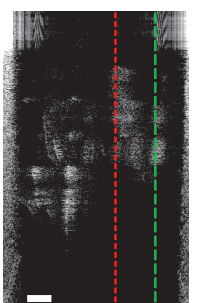

(c)
Fig. 3: Axial motion compensation in swept-source holographic OCT of glass beads rolled in a tape layer. The registered holograms are slices in the $(x, \omega)$ plane, for the $\mathrm{y}$ cut in Fig. 4. Figures represent the sample: (a) without any correction, (b) with interferogram rescaling (Eq. (2)), and (c) with interferogram rescaling and axial motion compensation (Section 3.2). The white scale bars indicate $0.2 \mathrm{~mm}$.

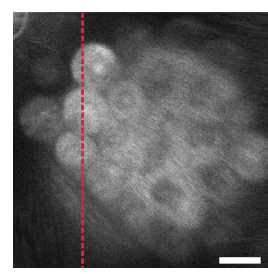

(a) Before processing

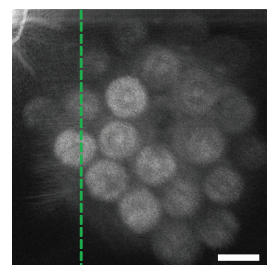

(c) Before processing

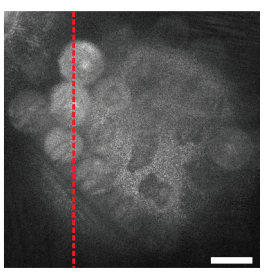

(b) After rescaling (Eq. (2))

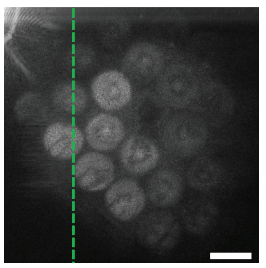

(d) Likewise (b)
Fig. 4: $(\mathrm{a}, \mathrm{b})$ En-face $(x, y)$ images of glass beads for the first red z-cut in Fig. 3, and (c,d) for the second green z-cut in Fig. 3. The white scale bars indicate $1.5 \mathrm{~mm}$.

tively. The quantitative comparison between $f_{1}$ and $f_{2}$ is performed by calculating cross-correlation whose maximum position gives a frequency shift $\Delta \omega\left(t_{0}^{\prime}\right)$ corresponding to the derivative of the phase caused by movement at time $t_{0}^{\prime}$. Axial motion compensation is then performed by substracting the cumulated phase shift $\int_{t_{0}^{\prime \prime}}^{t_{0}^{\prime}} \Delta \omega(t) d t$ from the phase $\phi\left(t_{0}^{\prime}\right)$. This procedure is repeated for all times $t_{0}^{\prime}$ in the time gate $g_{T}$ used in Eq. (4) of width $\omega_{\mathrm{S}} T /(2 \pi)=256$ points [15].

\section{RESULTS}

Fig. 2 shows retinal vessels acquired by laser Doppler imaging. Fig. $2 \mathrm{a}$ is the whole $2048 \times 2048$ image. The crossbeating interferometric contribution, which is the object of interest in the right bottom side of the image, is spatially separable from the other parts because of the off-axis configuration. Fig. $2 b$ shows the focus on retinal vessels. Ves- 
sels are moving in the image and sometimes disappear during several frames. Then, vessels do not appear clearly on the time-averaged image of 324 consecutive frames (Fig. 2c). After lateral stabilization, vessels are more visible on the timeaveraged image: motion compensation is efficient. Although motion compensation is less efficient when the image changes too often, it improves overall image quality.

Fig. 3 and Fig. 4 are swept-source OCT images of samples composed of $1.5 \mathrm{~mm}$ diameter glass beads rolled in a single tape layer. Fig. 3 shows the in-depth profile $(x, \omega)$ of this sample corresponding to the $y$-axis represented by dotted lines in Fig. 4. Fig. 4 shows the en-face $(x, y)$ images at two different depths corresponding to the dotted lines in Fig. 4. Fig. $4 \mathrm{a}$ and $4 \mathrm{~b}$ correspond to the red dotted line (depth of 1.1 $\mathrm{mm}$ ) in Fig. 3, and Fig. 4c and 4d correspond to the green dotted line (depth of $1.5 \mathrm{~mm}$ ) in Fig. 3. In Fig. $4 \mathrm{a}$ and $4 \mathrm{~b}$, the target layer is located between tape and beads: the beads on the left are at the same elevation as the tape layer on the right which starts to be sectioned.

A difference of lateral resolution is visible on images of Fig. 4. The interferogram rescaling allows the contours of the beads to be cleaner. Comparing Fig. 3a and Fig. 3b shows an improvement in axial resolution, which is also noticeable on en-face images: in Fig. 4c, beads on the right seem almost at the same elevation than beads on the left, because the depth accuracy is low, while in Fig. 4d, right beads clearly belong to a different layer. Axial motion correction can be seen in Fig. 3c: the accuracy is improving and the different layers are better separated. Besides, the optical bench will be strenghtened to reduce mechanical noise, in order to further improve the axial accuracy of depth images.

\section{CONCLUSION AND PROSPECTS}

We have demonstrated lateral and axial motion compensation in laser Doppler holography and holographic OCT by crosscorrelation stabilization, respectively. This method is suited to our images and cancels efficiently the effects of motion. Besides, the reported results are non-iterative and compatible with real-time processing at high throughput on graphics processing units. A real-time implementation of lateral motion compensation has been implemented ${ }^{2}$. The reported results in motion compensation pave the way towards the design of high resolution computational imaging for the retina in realtime by digital holography.

Aside from motion, retinal imaging suffers from phase distortions caused by the eye: the light backscattered by the retina crosses lenses in the eye, which can cause aberrations. As a future work, we will try to correct optical aberrations.

Funding: This work has been supported the European Research Council (ERC Synergy HELMHOLTZ, grant agreement \#610110).

\footnotetext{
${ }^{2}$ https: / / youtu.be/RhVPXBnPhXc
}

\section{References}

[1] Joseph L Calkins and Carl D Leonard, "Holographic recording of a retina using a continuous wave laser," Investigative Ophthalmology \& Visual Science, vol. 9, no. 6, pp. 458-462, 1970.

[2] R Lawrence Wiggins, K Diane Vaughan, and Gerhart B Friedmann, "Fundus camera holography of retinal microvasculature," Arch Ophthalmol, vol. 88, pp. 75-79, 1972.

[3] Manuel Simonutti et al., "Holographic laser doppler ophthalmoscopy," Optics letters, vol. 35, no. 12, pp. 1941-1943, 2010.

[4] Mathilde Pellizzari et al., "High speed optical holography of retinal blood flow," Optics letters, vol. 41, no. 15, pp. 35033506, 2016.

[5] Adolf F Fercher, Wolfgang Drexler, Christoph K Hitzenberger, and Theo Lasser, "Optical coherence tomography-principles and applications," Reports on progress in physics, vol. 66, no. 2, pp. 239, 2003.

[6] David Huang et al., "Optical coherence tomography," Science (New York, NY), vol. 254, no. 5035, pp. 1178, 1991.

[7] Caroline Magnain et al., "Holographic laser doppler imaging of microvascular blood flow," JOSA A, vol. 31, no. 12, pp. 2723-2735, 2014.

[8] Max A. Viergever et al., "A survey of medical image registration - under review," Medical Image Analysis, vol. 33, pp. 140-144, 2016.

[9] Chengyin Liu, Jiayi Ma, Yong Ma, and Jun Huang, "Retinal image registration via feature-guided gaussian mixture model," JOSA A, vol. 33, no. 7, pp. 1267-1276, 2016.

[10] Roziana Ramli et al., "Feature-based retinal image registration using d-saddle feature," Journal of healthcare engineering, vol. 2017, 2017.

[11] Nicolas Verrier and Michael Atlan, "Off-axis digital hologram reconstruction: some practical considerations," Applied optics, vol. 50, no. 34, pp. H136-H146, 2011.

[12] Pietro Ferraro et al., "Controlling image size as a function of distance and wavelength in fresnel-transform reconstruction of digital holograms," Optics Letters, vol. 29, no. 8, pp. 854-856, 2004.

[13] Vít Lédl, Pavel Psota, František Kaván, Ondřej Matoušek, and Pavel Mokrỳ, "Surface topography measurement by frequency sweeping digital holography," Applied optics, vol. 56, no. 28, pp. 7808-7814, 2017.

[14] Dirk Padfield, "Masked object registration in the Fourier domain," IEEE Transactions on Image Processing, vol. 21, no. 5, pp. 2706-2718, 2012.

[15] Dierck Hillmann et al., "Common approach for compensation of axial motion artifacts in swept-source OCT and dispersion in Fourier-domain OCT," Optics express, vol. 20, no. 6, pp. 6761-6776, 2012. 